REVISTA CHILENA DE NEUROPSICOLOGIAA UNIVERSIDAD DELAFRONTERA

\section{Revista Chilena de Neuropsicología}

ISSN: 0718-0551

editor@neurociencia.cl

Universidad de La Frontera

Chile

Solovieva, Yulia; García, Marco; Machinskaya, Regina; Quintanar, Luis Evaluación neuropsicológica y electrofisiológica en un adolescente autista y su corrección

Revista Chilena de Neuropsicología, vol. 7, núm. 2, 2012, pp. 91-97

Universidad de La Frontera

Temuco, Chile

Disponible en: http://www.redalyc.org/articulo.oa?id=179324185009

Cómo citar el artículo

- Número completo

- Más información del artículo

- Página de la revista en redalyc.org

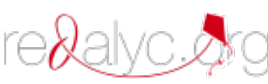

Sistema de Información Científica

Red de Revistas Científicas de América Latina, el Caribe, España y Portugal Proyecto académico sin fines de lucro, desarrollado bajo la iniciativa de acceso abierto 


\title{
Evaluación neuropsicológica y electrofisiológica en un adolescente autista y su corrección
}

Neuropsychological and electrophysiological assessment with correction of adolescent with autism

\author{
Yulia Solovieva ${ }^{1 *}$, Marco García ${ }^{1}$, Regina Machinskaya² ${ }^{2}$ Luis Quintanar ${ }^{1}$ \\ 1 Facultad de Psicología, Universidad Autónoma de Puebla. Puebla, México. \\ 2 Instituto de Fisiología del Desarrollo de la Academias de las Ciencias de Educación Rusa. Moscú, Rusia.
}

\section{Resumen}

El autismo es uno de los trastornos del desarrollo más complejos, tanto por la comprensión del cuadro clínico, su diagnóstico como y la falta de programas eficientes para el tratamiento. Una alternativa que está abordando este trastorno es la neuropsicología histórico-cultural. El objetivo es mostrar la eficacia de un programa de corrección que es formulado a partir de la evaluación neuropsicológica y la utilidad del electroencefalograma para tener un diagnóstico funcional claro en un paciente de 16 años con diagnóstico de autismo y retraso mental. El proceso de evaluación pre-y post se realizó con la metodología luriana, así como el programa de corrección. El análisis de los resultados fue intra-sujeto, diseño pre-experimental. El EEG se registró de acuerdo al sistema internacional 10-20 y para la conclusión se utilizó el esquema estructural para el análisis de fenómenos electrofisiológicos. La evaluación reveló insuficiencia funcional en los factores de regulación y control de la actividad voluntaria y en el análisis espacial simultáneo. El análisis cualitativo del electroencefalograma facilitó identificar la disfunción en los sistemas límbicos cerebrales regulatorios. El programa de corrección evidenció mejorías en todas las esferas del paciente. Se concluye que la metodología aplicada puede ser de utilidad para el tratamiento de autismo en la adolescencia.

Palabras clave: autismo, corrección neuropsicológica, electroencefalografía, evaluación neuropsicológica

\begin{abstract}
The autism is considered as one of the most complex disturbances in child development including both clinic assessment and organization of methods for intervention. Not all methods are efficient enough. Historic and cultural neuropsychology could be one of alternative proposals. The objective of our study is to show the efficiency of the Program for intervention formulated on the bases of neuropsychological assessment in a case of autism in the age of adolescence of 16 years old. Qualitative visual analysis of EEG was included as one of the methods for assessment of the patient. Clinical assessment and creation of the Program for intervention was designed according to Luria's methodology. The study presents the results of neuropsychological assessment before and after implementation of the program of intervention. The EEG was applied according to international system 10-20. Qualitative visual analysis of electrophysiological phenomenon was fulfilled. Neuropsychological assessment before the implementation of the program pointed out strong deficit of regulation and control of voluntary activity. The electrophysiological analysis detected severe disfunction of limbic subcortical regulation. The results of assessment alter correction showed significant improvement in activity and life of the patient. The proposed method could be useful in cases of autism in adolescence.
\end{abstract}

Keywords: autism, neuropsychological correction, electrophysiology, neuropsychological assessment

\footnotetext{
* Correspondencia: yulia.solovieva@correo.buap.mx. Facultad de Psicología, Universidad Autónoma de Puebla. 3 oriente 403, Puebla, Centro, México. Teléfono: + 52 (22) 012222425370

Recibido: 07-03-12. Revisión desde: 10-03-12. Aceptado: 25-05-12
}

DOI: $10.5839 / \mathrm{rcnp} .2012 .0702 .09$ 


\section{Introducción}

El estudio del cuadro o síndrome del autismo se inició con Kaner (1943) y Asperger (1944). Actualmente a partir de múltiples estudios, que siguieron a estos primeros, la APA y la OMS a través del DSM IV-TR (2000) y el CIE-10 (1992), respectivamente, han caracterizado el trastorno en tres síntomas principales: 1) la perturbación grave de habilidades para la interacción social, 2) de habilidades para la comunicación y 3) la presencia de comportamientos, intereses y actividades estereotipados.

Algunos autores refieren que el autismo puro no se explica por ninguna causa particular aparente, sin embargo, en la literatura actual se señala que es posible identificar alguna enfermedad que puede estar asociada al trastorno o que puede ser la causa del mismo (Artigas-Pallarés, Gabau-Vila, \& GuitartFeliubadaló, 2005).

Dentro de la concepción psicológica de la actividad humana es posible tratar de comprender el autismo como alteraciones del desarrollo en el sistema de organización afectiva de la conciencia, conducta y de su mecanismo principal, que es el sentido (Leontiev, 2000) y experiencias afectivas (Vigostky, 1999), las cuales determinan la visión del mundo e interacción en él.

Los estudios electrofisiológicos realizados a través de análisis de los electroencefalogramas de los pacientes con autismo refieren la existencia de cambios patológicos en la dinámica córtico-subcortical, con el predominio de problemas en las estructuras profundas medias límbicas, alteraciones a nivel de hipotálamo y diencéfalo, atrofias subcorticales y parietofrontales (Nurieva, 2003).

Dentro del sistema funcional de la acción humana los problemas de regulación límbico-frontal que se observan en los casos de autismo pueden significar problemas tanto a nivel subcortical como cortical, o de interacción entre ambos. Lo cual desde la perspectiva luriana (Luria, 1978) se refiere al primer bloque cerebral (problemas en regulación de incremento o decremento general y emocional de la actividad) y al tercero (formulación de motivos concientes, así como la valoración y verificación de todo el transcurso de la actividad).

Al afectarse desde edades muy tempranas la interacción de estos bloques funcionales, y lo que otros llaman como el "cerebro social" (estructuras frontales, temporales y subcorticales) (Brothers, 2003) habría, además de una percepción innadecuada de la conducta social, repercusiones graves en la conformación de las primeras neoformaciones psicológicas que serán vitales para la consolidación de la base del desarrollo de las siguientes edades, como por ejemplo, el inicio de una personalidad independiente que valora a su entorno de acuerdo a las relaciones emocionales con los adultos cercanos, al final del primer año de vida (Lisina, 1986).

Las propuestas para el diagnóstico y corrección del autismo, frecuentemente, no se relacionan con la posibilidad de conocer el estado funcional particular de las áreas cerebrales corticales y subcorticales con el fin de caracterizar y precisar las características negativas y positivas del cuadro. Este tipo de análisis lo puede garantizar solamente la evaluación neuropsicológica.

El objetivo del presente caso es mostrar la utilidad de la evaluación neuropsicológica en los casos de autismo para la elaboración y aplicación de estrategias interventivas, así como el gran apoyo del análisis cualitativo del electroencefalograma para el diagnóstico.

\section{Paciente}

Se trata de un adolescente masculino de 16 años de edad. La madre solicitó la evaluación neuropsicológica por dificultades en la comprensión y expresión verbal, problemas en la mayoría de sus materias escolares, además de dificultades de comunicación e interacción en general.

En la historia clínica del paciente se reporta uso de fórceps durante el parto, así como ausencia de actividad de juego en las edades tempranas y de dificultades en la interacción y comunicación con coetáneos. El paciente tuvo diagnósticos de autismo, retardo mental, retardo en adquisición del lenguaje y en el desarrollo psicomotor. El paciente cursa preparatoria abierta con apoyo pedagógico y cursos de computación y pintura. Un reporte de SPECT cerebral perfusorio (practicado a los once años de edad) refiere asimetría perfusoria en corteza cerebral por "menor perfusión en el lóbulo frontal izquierdo, sobre todo en los cortes inferiores. Los ganglios basales presentan perfusión discretamente asimétrica, por ser menor en el lado izquierdo", se concluye que las asimetrías no son extensas ni severas. El paciente fue medicado con melleril hasta los 5 años (con dos años de tratamiento) y posteriormente con tegretol (también dos años).

\section{Método}

El sujeto fue evaluado neuropsicológicamente de acuerdo a la metodología Luriana. Posteriormente se llevó a cabo un programa de intervención, por un periodo de casi 2 años una vez por semana (80 sesiones). El análisis de los resultados fue intrasujeto, diseño pre-experimental (pre-test/post-test). El éxito de la evaluación final se reporta en relación con los resultados de la evaluación inicial. El EEG se registró de acuerdo al sistema internacional 10-20 y para la conclusión se utilizó el esquema estructural.

\section{Resultados}

Durante la primera evaluación neuropsicológica el paciente presentó preocupación por realización de las tareas, aunque cooperador. Se observaron estereotipos conductuales que se manifestaron en observación exagerada de los detalles de vestimenta de personas ajenas a la actividad de evaluación.

Para la Evaluación Neuropsicológica inicial y final se utilizaron instrumentos diseñados por Quintanar y Solovieva (2003).

La evaluación neuropsicológica inicial evidenció la conservación de los factores que garantizan y regulan la comprensión de los sonidos del lenguaje (oído fonemático), del reconocimiento cinestésico-táctil, de la retención visual y la conformación de la imagen objetal. Las debilidades funcionales de la evaluación inicial pueden observarse en la tabla 1.

\section{Programa}

El programa de corrección tuvo como objetivo reorganizar los mecanismos neuropsicológicos que evidenciaron insuficiencia funcional. El programa se formuló con base en los principios 
Tabla 1. Cambios observados en los factores neuropsicológicos débiles.

\begin{tabular}{|c|c|c|}
\hline Variable & Evaluación Inicial & Evaluación final \\
\hline \multirow{2}{*}{$\begin{array}{l}\text { Regulación y control de la } \\
\text { actividad }\end{array}$} & \multirow{2}{*}{$\begin{array}{l}\text { Pérdida del objetivo en lenguaje, escritura, lectura y cálculo así como } \\
\text { imposibilidad de verificación y corrección por su impulsividad }\end{array}$} & Reducción considerable de la impulsividad \\
\hline & & $\begin{array}{l}\text { Considera el objetivo de la actividad al repetir o al pedir la instrucción } \\
\text { dada }\end{array}$ \\
\hline \multirow[t]{2}{*}{ Melodía cinética } & Cierta lentitud en la secuencia gráfica & Mejoría en fluidez \\
\hline & Frecuentes agramatismo verbales & Disminución de los agramatismos \\
\hline $\begin{array}{l}\text { Análisis y síntesis espacial } \\
\text { simultáneo }\end{array}$ & $\begin{array}{l}\text { Problemas de expresión y comprensión de estructuras genitivas, } \\
\text { comparativas, inversas, pasivas, espaciales y temporales }\end{array}$ & $\begin{array}{l}\text { Mejoría en la comprensión de este tipo de oraciones pero únicamente } \\
\text { cuando hay apoyo de objetos concretos }\end{array}$ \\
\hline Retención audio-verbal & $\begin{array}{l}\text { Involuntaria y voluntaria: dificultades desde la repetición y disminu- } \\
\text { ción en volumen en la evocación ( } 3 \text { de } 6 \text { y } 4 \text { de } 6 \text { respectivamente, } \\
\text { aumentando palabras no contenidas en la serie }\end{array}$ & $\begin{array}{l}\text { Evocó el mismo número de palabras a nivel involuntario. A nivel } \\
\text { voluntario evocó una palabra más. Lo evidente fue la mejoría en los } \\
\text { procesos inhibitorios puesto que ya no hubo asociaciones secundarias }\end{array}$ \\
\hline Retención visuo-motora & $\begin{array}{l}\text { Reducción en el volumen de información ( } 3 \text { de } 6 \text { ). Y contaminación de } \\
\text { elementos }\end{array}$ & $\begin{array}{l}\text { Aumento en el volumen de información evocada ( } 5 \text { de } 6 \text { ) y sin errores } \\
\text { de contaminación }\end{array}$ \\
\hline
\end{tabular}

teórico -metodológicos de la escuela histórico-cultural (Luria, 1998; Tsvetkova, 1977; Solovieva, Bonilla, \& Quintanar, 2007).

Las actividades del programa de corrección neuropsicológica se dirigieron a los objetivos de consolidación de regulación y control de la actividad propia del paciente y de análisis y síntesis espaciales en los niveles material, perceptivo y verbal.

Es importante hacer mención que únicamente se realizó la primera etapa del programa por situaciones de la familia. Se consideró la participación de la madre del paciente la cual fue de gran apoyo (Mulas, Ros-Cervera, Millá, Etchepareborda, Abad, \& Téllez, 2010).

A continuación se mostrarán algunas tareas llevadas a cabo.

Trabajo con el factor de regulación y control

a) Tablas de Shults: el paciente elabora una tabla del 100 al 200 al azar. Y posteriormente tiene que ir señalando los números de acuerdo a algún orden específico.

b) Trabajo con la lectura de textos.

- el paciente recibe un texto literario.

- $\quad$ el paciente lee una palabra en silencio, una en voz alta, una en silencio, dos en voz alta, tres en silencio, una en voz alta, etc.

- $\quad$ el paciente lee todo el texto en silencio.

- $\quad$ el paciente lee todo el texto en voz alta.

c) Solución de problemas intelectuales.

- juegos de selección: ante palabras específicas el paciente realiza movimientos determinados.

- juegos de mesa con orientación del psicólogo.

- enunciar una palabra de manera alternada: la palabra que se menciona debe empezar con el mismo sonido con el cual termina la anterior.

d) En la mayoría de las tareas se escribía la instrucción y el paciente tenía que leerla y expresarla para que realizara lo que se le indicaba.

Trabajo con el factor de análisis y síntesis espaciales simultáneas

a) Construcción y seguimiento de frases con nociones espaciales (a, hacia, entre, arriba, abajo, enfrente, atrás, sobre, etc.) con material concreto.
- Se trabaja con un objeto con diferentes preposiciones y una misma preposición con diferentes objetos.

- Se trabaja con la preposición "de" en su sentido genitivo ayudado por esquemas de familiares.

- El psicólogo y el paciente salen a la calle a algún lugar conocido (parque, juegos y oficinas) y, en el camino se comentan detalles que se observan, al regreso el paciente hace el recorrido con palitos sobre la mesa, posteriormente se hace la misma actividad pero ahora representa el camino en un esquema gráfico.

b) Cuando ya se hubo trabajado con la estructura de la oración, el psicólogo realiza acciones y el paciente tiene que describir las mismas y anotarlas.

c) De manera conjunta, por medio de preguntas se le ayuda al paciente a que identifique acciones en cuadros temáticos.

d) Identificación y expresión de emociones.

e) Trabajo con cuentos apoyados de la imagen, con el objetivo de realizar planes y mejorar su actividad intelectual y su lenguaje.

f) Dictado gráfico: se le indica al paciente en que dirección tiene que hacer líneas de tal manera que se obtengan figuras geométricas.

\section{Trabajo con el lenguaje expresivo}

a) Trabajo con el esquema lineal de la oración. Tiene como objetivo que el paciente estableciera las relaciones entre los miembros gramaticales de una oración y visualizara de manera concreta la sintaxis de una oración simple:

Se proporcionan tarjetas orientadoras (una para cada miembro) que explican brevemente los diferentes miembros gramaticales y se concluye con la pregunta o preguntas que apoyan la identificación de los mismos.

b) Se le proporciona oraciones en las cuales tiene que identificar los diferentes miembros gramaticales de la oración, subrayando con un color específico cada uno de los miembros.

c) El psicólogo hace acciones y el paciente tiene que elaborar la oración con las tarjetas orientadoras. Posteriormente, se eliminan las tarjetas orientadoras y se le proporcionan tarjetas blancas, las cuales ira colocando de acuerdo a cada elemento de la oración que vaya formulando. 
Figura 1. Pinturas al óleo antes y después de la corrección.
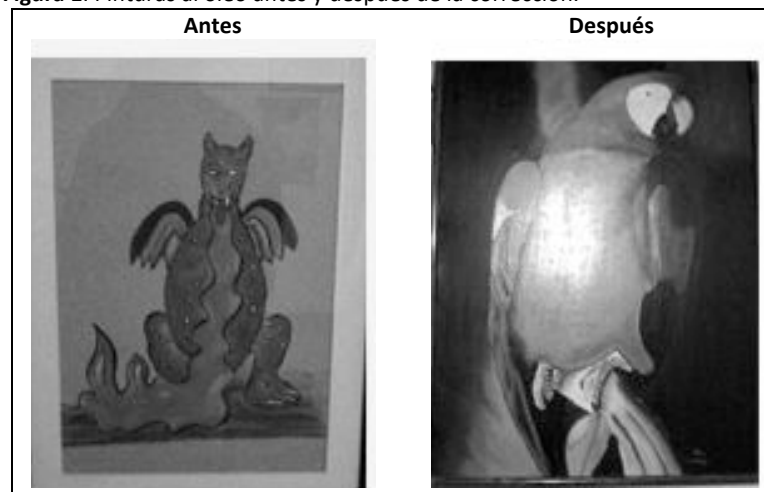

Nota. Obsérvense mejorías en la actividad gráfica del paciente: en la perspectiva, las proporciones y en general en la calidad de la pintura. Fotografía: Marco García.

Figura 2. Copia de una casa antes y después de la corrección.

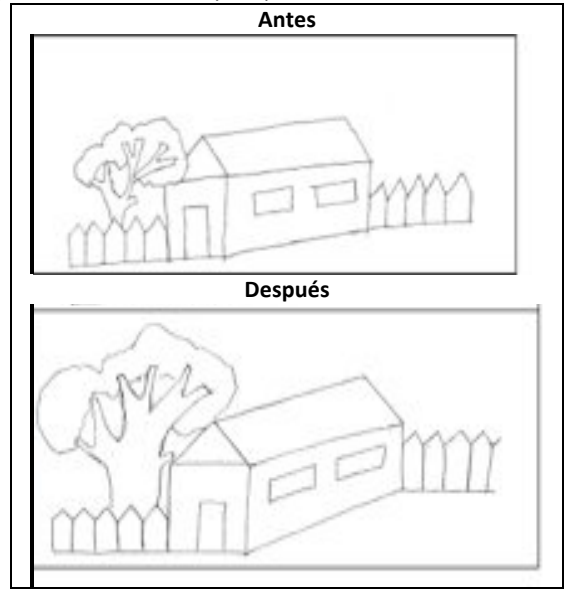

Nota. Existe una mejor representación de la profundidad. Fotografía: Marco García.
Figura 3. Formulación de una oración antes y después de la corrección, ante una fotografía.

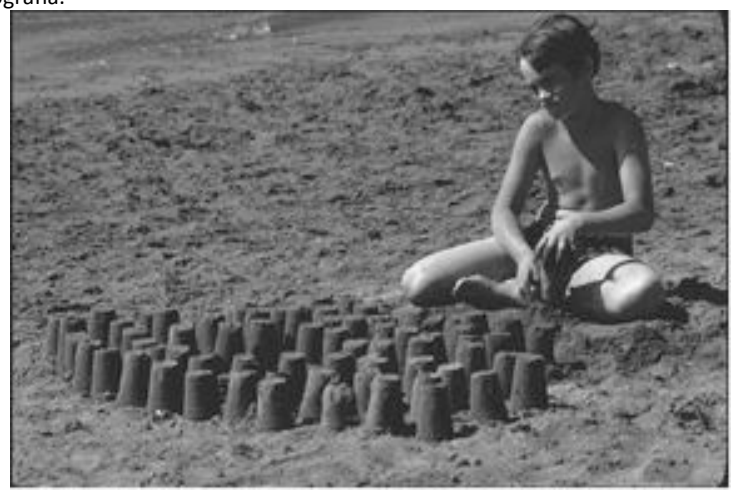

Nota. Antes: "el niño construyendo en la arena de piloncillo para hacer un dulce de calabaza". Después: "el niño está haciendo con vasos arena castillos". Fotografía: Marco García.

Figura 4. Comparación de cuadros temáticos con el mismo sentido y diferente significado.
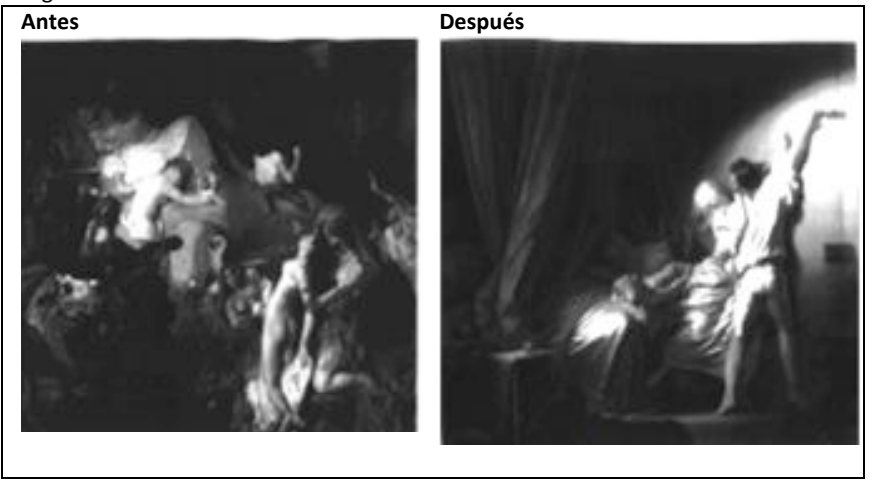

Nota. “¿Dónde está representada la emoción de agresividad?" Antes: responde impulsivamente señalando el primero y refiere: "está actuando en emperador en el siglo XXI en 1806". Después: realiza un adecuado rastreo visual y señala correctamente. Fotografía: Marco García.

Figura 5. Actividad electroencefalográfica que evidencia cambios en la actividad eléctrica de origen límbico.

\begin{tabular}{|c|c|}
\hline $02-12$ & 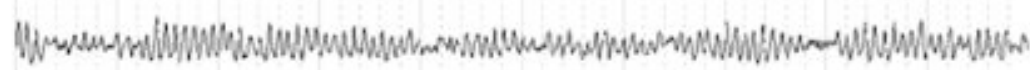 \\
\hline PA.A2 & 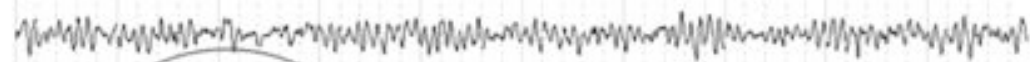 \\
\hline$a \cdot A_{2}$ & 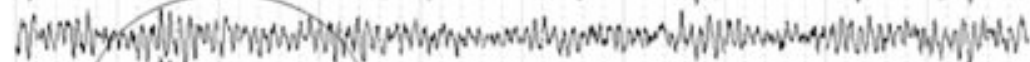 \\
\hline $\mathrm{F} 4 \cdot \mathrm{A}^{2}$ & 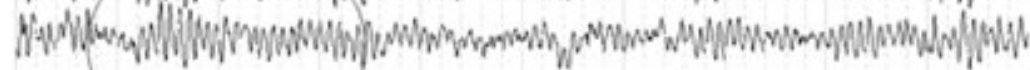 \\
\hline TAA2 & 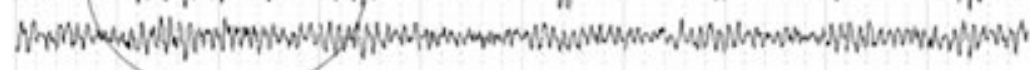 \\
\hline T6:A2 & 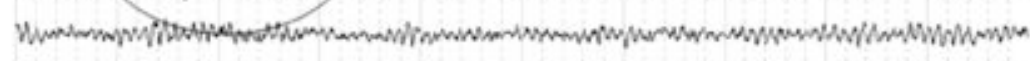 \\
\hline 01-A1 & 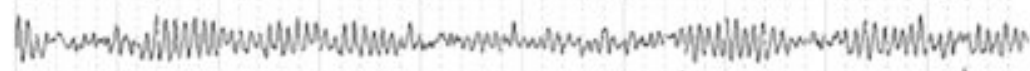 \\
\hline P3.At & 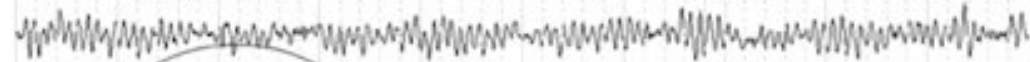 \\
\hline C3.At & aWhW W \\
\hline F):A1 & 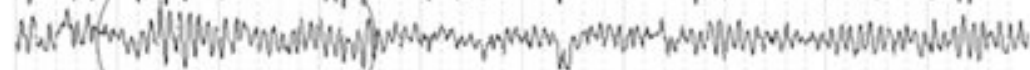 \\
\hline that & nWur \\
\hline TSAA & 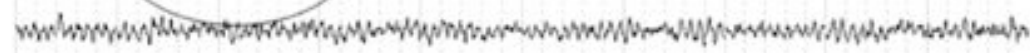 \\
\hline
\end{tabular}

Nota. Se observan oscilaciones en forma de husos bilaterales sincronizados con la frecuencia de $11 \mathrm{~Hz}$ en los sectores centrales $\left(\mathrm{C}_{3,4}\right)$, frontales $\left(\mathrm{F}_{3,4}\right)$ y temporales anteriores $\left(T_{3,4}\right)$. La amplitud máxima de estos husos se registraba en los sectores frontales y superaba dos veces la amplitud del ritmo de fondo registrado en los sectores occipitales. 
En la tabla 1 se pueden observar los cambios mostrados después de la corrección neuropsicológica .

Las mejorías en los diversos factores neuropsicológicos tuvieron un efecto sistémico en la actividad gráfica (ver figuras 1 y 2 ), en el lenguaje (ver figura 3), y en la actividad intelectual (ver figura 4).

En cuanto al lenguaje, los estereotipos verbales que mostraba en un inicio los fue cambiando por frases que le ayudaban a automotivarse y a recibir retroalimentación por parte del terapeuta: "verdad que ya hago mejor las cosas", o antes de contestar una pregunta refiere "analizar la pregunta" y/o "primero sujeto, después verbo y después complemento". Actualmente ya puede llevar a cabo diálogos cortos y seguir convencionalismos sociales (saludar, despedirse, pedir permiso, etc.). Cabe mencionar que todavía le falta interiorizar las ayudas que él mismo emite con su lenguaje externo y consolidar la estructura de las oraciones, como se observa en la figura 3.

En cuanto a la actividad intelectual en la evaluación inicial, el paciente tuvo dificultades en las operaciones de análisis y síntesis, tanto en textos como en cuadros artísticos. Actualmente, ha mejorado la operación de análisis (aunque no de síntesis) y en la inhibición de ideas no contenidas en los textos.

Hubo mejorías en la identificación de estados emocionales, por medio de la adecuada percepción de las caras.

En la expresión del lenguaje, había dificultades en la formulación de oraciones, actualmente ha mejorado este aspecto (aunque todavía falta su consolidación).

Cabe mencionar que actualmente el paciente lleva una vida muy independiente, con supervisión de la madre, pues se traslada solo de un lugar a otro en el trasporte público e ingresó a un trabajo el cual es remunerado, además de que ha empezado a vender sus obras de arte (pintura al óleo).

Para el registro de EEG se utilizó el equipo computarizado Nicolet (Estados Unidos). El EEG se registró desde los sectores $\mathrm{O}_{1,2} ; \mathrm{P}_{3,4} ; \mathrm{T}_{6,5} ; \mathrm{T}_{3,4} ; \mathrm{C}_{3,4} ; \mathrm{F}_{3,4}$, en correspondencia con el sistema internacional $10-20 \%$ con los electrodos auriculares ipsilaterales. Para el análisis se aplicó el montaje mono y bipolar.

Para la descripción de los patrones de EEG y la obtención de la conclusión se utilizó el esquema estructural (Luckashevi, Machinskaya, \& Fishman,1999), el cual permite valorar el estado de la corteza en general y de sus sectores particulares, así como la presencia de desviaciones de la actividad eléctrica en las estructuras regulatorias profundas subcorticales de diversos niveles.

El análisis visual de EEG indicó que en general el estado de las estructuras ritmógenas corticales, que fue valorado de acuerdo a la frecuencia, regularidad y reactividad hacía la fotoestimulación e hiperventilación del ritmo básico de EEG corresponde a la norma de la edad. Se registró ritmo alfa modular con la frecuencia de $10-11 \mathrm{~Hz}$ y amplitud de 30 a $70 \mathrm{Mkv}$., el cual predominaba en los sectores occipitales. En el EEG no se observó ningún tipo de la actividad desviada en los sectores de la corteza ni en el estado de reposo, ni tampoco ante la aplicación de las pruebas funcionales (hiperventilación y fotoestimulación). Tampoco se detectaron cambios del tipo paroxístico en su EEG. La particularidad clínica de este paciente puede observarse en la figura 5.

\section{Discusión}

Muchos investigadores consideran que es primordial ahondar sobre la comprensión del Autismo, más que encontrar una cura. Esta manera de ver al autismo se debe a que en sus marcos de referencia, el conocer la etiología orgánica que sufrió el individuo en alguna etapa del desarrollo o la identificación de un marcador biológico (Artigas-Pallarés, Gabau-Vila \& GuitartFeliubadaló, 2005; Tuchman, 2001; Alessandri, Thorp, Mundy, \& Tuchmanet, 2005) es condición necesaria para entender la naturaleza del mismo. Por lo tanto, la intervención tanto curativa como preventiva será primordialmente a nivel orgánico, a pesar de que se reconoce el abordaje interdisciplinario.

A pesar de tener estas consideraciones, la mayoría de los que han abordado el trastorno autista basan su diagnóstico en listas de observación con criterios tomados del DSM-IV-TR o CIE-10 y pruebas estandarizadas (Posada, 2005).

Por otro lado, la aproximación neuropsicológica, además de considerar como importante los antecedentes biopatológicos del trastorno, el objetivo primordial de la evaluación es identificar el estado funcional actual de los factores neuropsicológicos, los cuales son el substrato psicofisiológico de la actividad mental y por ende la causa que explica los síntomas. En otras palabras, sea cual fuese la causa orgánica del trastorno autista que aconteció en la historia del desarrollo del sujeto (genéticas, morfológicas, metabólicas, traumáticas, infecciosas, etc.) y que ha dejado sus secuelas en el sistema nervioso, no nos queda mejor opción que descubrir en el presente el síndrome neuropsicológico. Consiste en identificar el mecanismo cerebral que se encuentra con alguna insuficiencia funcional, y qué funciones psicológicas están alteradas como consecuencia de esta afectación y cuáles están conservadas (análisis sindrómico) y a partir de ello diseñar una metódica específica de corrección.

Por lo tanto la evaluación neuropsicológica posibilita conocer la causa funcional que subyace a los síntomas presentados por el paciente, y no solamente realiza una descripción fenomenología del trastorno. Vigotsky (1999), ya refería que los métodos de evaluación que llevan a cabo una "suma mecánica de síntomas, en sí misma, no pueda dar un diagnóstico científico" (pag. 303). Además de que se explora la zona de desarrollo próximo (Solovieva \& Quintanar, 2004) y el proceso de solución de la tarea, con el objetivo de identificar que tipo de ayudas le son adecuadas para resolver las tareas planteadas y la manera en que el paciente logra el resultado (Vigotsky,1999; Solovieva \& Quintanar, 2004).

En el caso de nuestro paciente los factores que evidenciaron insuficiencia funcional fueron el de regulación y control de la actividad conciente (sobre todo en la regulación de estados emocionales) y en el análisis y síntesis espacial simultánea (zonas terciarias del hemisferio izquierdo).

Este diagnóstico funcional concordó con los estudios de SPECT y electroencefalograma que le fueron aplicados; el primero refería que había asimetría perfusoria en corteza cerebral, observándose menor perfusión en el lóbulo frontal izquierdo, sobre todo en los cortes inferiores; el segundo estudio reportó cambios en la actividad eléctrica de origen límbico. A favor de esta interpretación hablan algunos datos diversas investigaciones. 
En primer lugar, en los estudios de la actividad neuronal en animales se ha mostrado que en las neuronas de la amígdala se generan las descargas rítmicas de grupos de oscilaciones con la frecuencia cercana a la frecuencia del ritmo alfa de 5-12 Hz (Schulz, Knuepfer, Lambertz, Langhorst, \&Stock.)

En segundo lugar, la expresividad de la actividad alfa en los EEG de los adultos sanos correlaciona positivamente con el nivel de metabolismo en las estructuras profundas del cerebro, entre otras, de la amígdala (Sadato, Nakamura, Oohashi, Nishina, Fuwamoto, Waki \& Yonekura, 1998).

En tercer lugar, ante el registro simultáneo del EEG desde la corteza cerebral y desde las estructuras profundas en el ser humano fue descubierto que los husos alfa, los cuales se generan en la corteza en el estado de sueño, surgen, inicialmente, en las estructuras límbicas; además, estos también se pueden registrar en estas mismas estructuras en el estado de vigilia (Caderas, Niedermeyer, Uematsu, Long \& Nastalski, 1982). En el caso de combinación del registro de EEG con resonancia magnética (MRI) con ayuda del método de localización de las fuentes de dipoles de la actividad eléctrica se encontró que la fuente de la actividad eléctrica del tipo alfa en forma de husos que se manifiesta en el grado máximo en los sectores frontales de la corteza, en los sujetos sanos durante el sueño se encuentra en el sistema límbico en la corteza singular (Menendez, Morier, Picard, Landis \& Gonzalez, 2006). A pesar del hecho de que en el EEG del adulto sano la actividad eléctrica cerebral del tipo alfa en forma de husos surge predominantemente durante el sueño, en el caso de nuestro paciente, este patrón de la actividad eléctrica cerebral se registró en el estado de vigilia. Sobre este hecho nos habla el fondo, sobre el cual se registró este patrón (ver figura 5). Así, estaban totalmente ausentes las oscilaciones lentas y otros patrones típicos para el estado de sueño. Además, frecuentemente, este patrón surgía de inmediato después de fotoestimulación, lo cual excluye la posibilidad del estado de sueño repentino.

Al tener claro el síndrome, el objetivo terapéutico estuvo encaminado a reorganizar el funcionamiento de los mecanismos psicofisiológicos débiles (regulación y control de la actividad voluntaria y análisis y síntesis espacial simultáneo). Fue fundamental en este trabajo la reorganización extracerebral mediatizada que componen las acciones que incluyen los mecanismos neuropsicológicos afectados; iniciando en el plano externo, con ayudas desplegadas, materializadas y en forma colaborativa. La finalidad fue lograr la interiorización gradual de dichas acciones, retirando paulatinamente las ayudas y los medios externos (Solovieva, Bonilla, \& Quintanar, 2007).

Con el trabajo correctivo se observaron ciertas mejorías, pero no la reorganización total de los mecanismos cerebrales afectados del paciente, debido a que únicamente se llegó a la fase material, iniciándose la perceptiva concreta. La reorganización parcial de estos factores neuropsicológicos tuvo un efecto sistémico en las diversas funciones psicológicas en los cuales participan (ver tabla 1, columna derecha). El paciente tuvo importantes mejorías en sus funciones ejecutivas (inhibición, planeación, verificación y corrección de su actividad), en su actividad intelectual, en la comprensión y expresión del lenguaje, en la lectura y escritura, así como en el reconocimiento de emociones por medio del análisis de los gestos faciales. Cabe mencionar que todavía faltaron etapas de corrección para evidenciar mayores logros en sus sistemas de actividad, como lo es en la mejora de la regulación emocional ante situaciones de exigencia social; en la completa interiorización tanto de la estructura de la morfo-sintaxis de su expresión verbal como en la comprensión de diversas y variadas oraciones complejas; en la solución de problemas y en una mayor flexibilidad y dinámica de su pensamiento.

\section{Conclusiones}

1. La evaluación neuropsicológica y el análisis del esquema estructural del electroencefalograma en un caso de autismo identificó que las principales alteraciones se observan en el inadecuado funcionamiento de zonas terciarias del hemisferio izquierdo y en las relaciones funcionales del tercer bloque funcional con el primero (principalmente a nivel límbico).

2. La evaluación neuropsicológica desde la perspectiva histórico-cultural posibilitó diseñar un programa dirigido a la causa (factores neuropsicológicos débiles) el cual tuvo efectos sistémicos en la comprensión y expresión del lenguaje, actividad gráfica, actividad intelectual, lectura e interacción social.

3. En el programa de corrección debe considerarse la interiorización progresiva de las acciones, partiendo del desarroIlo intelectual en que se encuentre el paciente.

4. El apoyo y el trabajo familiar es fundamental para el seguimiento del programa

5. El trabajo correctivo demostró que es posible observar mejorías a través de un programa de intervención neuropsicológica en pacientes adultos con trastorno autista

\section{Referencias}

Alessandri, M., Thorp, D., Mundy, P., \& Tuchmanet, R. F. (2005). ¿Podemos curar el autismo? Del desenlace clínico la intervención. Revista de Neurología, 40(supl 1), S131-S136.

Artigas-Pallarés, J., Gabau-Vila, E. \& Guitart-Feliubadaló, M. (2005). El autismo sindrómico: I. Aspectos generales. Revista de Neurología, 40(supl 1), S143S149.

Asociación Psiquiátrica Americana (2000). Manual Diagnóstico y Estadístico de los Transtornos Mentales. Cuarta edición, Texto revisado (DSM-IV-TR). España: Masson.

Asperger, H. D. (1944). Autistischsn Psychopathen im Kindesalter. Archiv. für Psychiatrie und Nervenkrankenheiten, 117. Versión inglesa en Frith, U. Autism and Asperger Syndrome.Cambridge: Cambridge University Press 1991.

Brothers, L. (2003).The social brain: A project for integrating primate behavior and neurophysiology in a new domain. In Mundy P. Annotation: The neural basis of social impairments in autism: the role of the dorsal medial-frontal cortex and anterior cingulated system. Journal of Child Psychology and Psychiatry, 44(6), 793-809.

Caderas, M., Niedermeyer, E., Uematsu, S., Long, D.M. \& Nastalski, J. (1982). Sleep spindles recorded from deep cerebral structures in man. Clinical Electroencephalogry, 13(4), 216-25.

O.M.S. (1992). CIE-10. Clasificación estadística internacional de enfermedades y problemas de salud Ginebra: Organización Mundial de la Salud.

Kanner, L. (1943). Autistic disturbances of affective contact. The Nervous Child, 2, 217-50.

Leontiev, A.N. (2000). Conferencias sobre psicología general. Moscú: Sentido.

Lisina, M. (1986). La comunicación con los adultos en los niños hasta los siete años de vida. En Iliasov I.I., y Liadus V.Y. (Ed.), Antología de la psicología pedagógica y de las edades (pp. 125-131). La Habana: Pueblo y Educación.

Luckashevi, I.P., Machinskaya, R.I., \& Fishman, M.N (1999). The EEG-EXPERT, Automatic Diagnostic System. Biomedical Engineering, 33(6), 302-330.

Luria, A. R. (1978). El cerebro en acción. Barcelona: Fontanella.

Luria, A. R. (1998). Rehabilitación de funciones cerebrales después de traumatismos de guerra. En Quintanar L, (comp). Problemas Teóricos y metodológicos de la rehabilitación (43-94). Tlaxcala: Universidad Autónoma de Tlaxcala.

Menendez, R., Morier, P., Picard, F., Landis, T., \& Gonzalez, A. S. (2006). Simple techniques for EEG source imaging. International Journal of Bioelectromagnetism, 8(1), 1-8.

Mulas, F., Ros-Cervera, G., Millá, M., Etchepareborda, M.C., Abad, L., \& Téllez, M. (2010). Modelos de intervención en niños con autismo. Revista de Neurología, 50(Supl 3), S77-S84. 
Nurieva, L.G. (2003). Desarrollo del lenguaje en niños con autismo. Moscú: Terevinf.

Posada, M. (2005). Investigación epidemiológica en el autismo: una visión integradora. Revista de Neurología, 40(Supl 1), S191-S198s.

Quintanar, L. \& Solovieva, Y. (2003). Manual de evaluación neuropsicológica infantil. México: Universidad Autónoma de Puebla.

Sadato, N., Nakamura, S., Oohashi, T., Nishina, E., Fuwamoto, Y., Waki, A., \& Yonekura, Y. (1998). Neural networks for generation and suppression of alpha rhythm: a PET study. Neuroreport, 9(5), 893-7.

Schulz, G., Knuepfer, M., Lambertz, M., Langhorst, P., \& Stock, G. (1996). Relationship between rhythmic discharge patterns of neurons in the central nucleus of the amygdala, blood pressure fluctuations and cortical activity. J. Auton Nerv Syst, 57(3), 158-62.

Solovieva, Y., Bonilla, R. \& Quintanar, L. (2007). Aproximación Histórico-Cultural: Intervención en los trastornos del aprendizaje. En Eslava C, Mejía L, (Ed.), Los trastornos del aprendizaje. Perspectivas neuropsicológicas (227-266). Bogotá: Cooperativa Editorial Magisterio, Instituto Colombiano de Neurociencias, Benemérita Universidad Autónoma de Puebla.

Solovieva, Y. \& Quintanar, L. (2004). La zona de desarrollo próximo como método para el diagnóstico del desarrollo intelectual. En: Castañeda F. (ed.), Educación aprendizaje y cognición (75-92). México: Manual Moderno.

Tsvetkova, L.S. (1977). Reeducación del lenguaje, la lectura y la escritura. Bacelona: Fontanela.

Tuchman, R. (2001). Como construir un cerebro social: lo que nos enseña el autismo. Revista de Neurología, 33(3), 292-299.

Vigotsky, L. S. (1999). Obras escogidas, Tomo IV. Madrid: Visor. 